\title{
SUSTENTABILIDADE ENERGÉTICA \\ EM ESCOLA PÚBLICA
}

\author{
ENERGY SUSTAINABILITY IN PUBLIC SCHOOL
}

\author{
MARI AURORA FAVERO REIS, Dra. | UnC \\ PAULO REIS JUNIOR, M.SC. UnC \\ DIRCEU LORIVALDO PERIN $\mid$ UnC
}

\begin{abstract}
RESUMO
Estabelecimentos de ensino públicos e privados apresentam-se como cenários oportunos para a busca de sustentabilidade energética. No Brasil, a tecnologia fotovoltaica se destaca devido aos índices elevados de radiação e a possibilidade de integrar com a rede elétrica tradicional. Uma pesquisa foi realizada com o objetivo de avaliar a viabilidade técnica e econômica para a instalação de painéis solares fotovoltaicos em dois estabelecimentos de ensino em Concórdia (SC). Para os cálculos foram considerados valores orçamentários junto a fornecedor da tecnologia e dados coletados nas edificações em estudo. Foi relacionado consumo de eletricidade nas edificações e condições físicas dos estabelecimentos, como possível posição, disposição dos painéis na cobertura e viabilidade econômica. Os valores de investimentos iniciais apresentados por fornecedor da tecnologia equacionaram resultados semelhantes, para retorno financeiro após quatro anos de instalação, com maior viabilidade na edificação de maior consumo. Com o estudo concluímos que o empreendimento de maior consumo de energia elétrica, com grandes áreas de cobertura, demonstrou ser mais viável para o uso dessa tecnologia.
\end{abstract}

PALAVRAS CHAVES: Fotovoltaica. Sustentabilidade energética. Viabilidade. Escola pública.

\begin{abstract}
Public and private schools that present opportunities for the pursuit of energy sustainability. In Brazil, photovoltaic technology is reduced due to high pollution rates and the possibility of integrating with the traditional grid. A research was carried out to evaluate the technical and economic feasibility for the installation of photovoltaic solar panels in two educational establishments in Concordia (SC). For the calculations were considered budget values with the technology supplier and the data collected in the buildings under study. It was related to the consumption of electricity in the buildings and physical conditions of the establishments, such as possible positioning, layout of the panels in the coverage and economic viability. The initial investment values presented by the technology provider equated similar results, for financial return after four years of installation, with greater viability in the construction of higher consumption. With the study we conclude that in the undertaking of higher consumption of electricity, with large areas of coverage, proved to be more viable for the use of this technology.
\end{abstract}

KEY WORDS: Photovoltaic. Energy sustainability. Viability. Public school. 


\section{INTRODUÇÃO}

Com o advento das perspectivas econômicas e ambientais vinculadas à economia circular, a energia solar fotovoltaica tem sido considerada fonte promissora na produção de energia, possibilitando a transformação da luz em eletricidade, atraindo os estudantes de Engenharia para o estudo da tecnologia. A justificativa de muitos autores para o uso da energia solar diz respeito ao fato de ser infinita e renovável (KORHONEN; HONKASALO; SEPPÄLÄ, 2018). Os autores consideram o fato de que a Terra recebe um fluxo de energia solar infinito e renovável que poderia, em teoria, ser aproveitado e utilizado para o aquecimento de água e produção de corrente, como é comumente realizado por intermédio de tecnologias fotovoltaicas nas células de silício.

Em 2015, ano em que a comunidade científica celebrava o Ano Internacional da Luz (AIL), nas comemorações dos 110 anos do artigo sobre efeito fotoelétrico publicado por Albert Einstein, iniciava-se uma pesquisa bibliográfica para construção de tese de doutorado (REIS, 2019), na qual as pesquisas analisadas na revisão sugeriam o uso da tecnologia fotovoltaica para explorar o quantum de luz (REIS; SERRANO, 2017). O uso da tecnologia como aplicação de contexto para o estudo na transformação da luz junto à Engenharia Civil motivou acadêmicos à exploração do tema em suas pesquisas de Trabalho de Conclusão de Curso para a obtenção do título de Bacharel em Engenharia Civil, como no caso do presente artigo.

$O$ interesse na escolha do ambiente de estudo como aplicação de contexto para pesquisa junto à Engenharia Civil teve origem em 2018, durante uma atividade de extensão universitária. Na ocasião, a Universidade oportunizou uma oficina com o uso de células fotovoltaicas para estudantes finais de Ensino Médio, em escolas públicas de Concórdia, SC. O professor de uma das escolas era professor de Matemática e acadêmico da Engenharia Civil, que identificou nos estudantes o interesse no estudo da temática. A oficina foi realizada com a integração da Ciência, Tecnologia, Engenharia e Matemática (STEM). O movimento STEM (do inglês Science, Technology, Engineering and Mathematics) tem foco na educação para o desenvolvimento de competências na liderança global do conhecimento, tendo como aplicação de contexto a tecnologia fotovoltaica (DARK, 2011).

A exploração de energia solar fotovoltaica neste tipo de edificação (escola) poderá motivar os estudantes para atitudes que promovam a sustentabilidade energética, além de contribuir para a disseminação da tecnologia. Para a sustentabilidade energética dos estabelecimentos educacionais, em que a demanda é elevada durante o período diurno, demostra ser apropriada a geração fotovoltaica. A efetivação desse cenário requer o desenvolvimento de competências nos métodos de produção de energia fotovoltaica, a fim de suprir a demanda para o futuro com investimentos pelo setor público estadual.

\section{REVISÃO BIBLIOGRÁFICA}

O cenário de oferta futura de energia elétrica para os próximos anos, projeções da demanda apontam para a fragilidade no sistema de energia no Brasil, mesmo considerando que o Brasil possui bom potencial hidrelétrico (SALAMONI; RÜTHER; ZILLES, 2009). Segundo os autores, o Brasil ainda possui um alto déficit em relação ao alcance da distribuição de energia elétrica. Nesse caso, a solução possível e em expansão é a instalação de sistemas de geração de energia a partir da radiação solar, na modalidade on-grid.

Em 2018, no Brasil, houve uma expansão em projetos de engenharia com o uso de energia solar fotovoltaica conectada à matriz elétrica. Ademais, países, como Alemanha, Estados Unidos, Espanha, França, Itália e Japão desenvolveram mecanismos que reduzem a dependência de combustíveis fósseis, introduzindo opções renováveis em seus suprimentos de energia primária e descentralizando a geração de energia eólica e biomassa (CARVALHO; ABREU; CORREIA NETO, 2017).

A Resolução Normativa n. 482/2012, da Aneel, reporta-se a sistemas de mini e microgeração conectados à rede de distribuição elétrica, o que possibilita aos consumidores autogerarem energia elétrica para consumo próprio, mediante mecanismo de compensação (SOUZA; MACHADO, 2016). Essa possibilidade contribuiu para a expansão da matriz elétrica fotovoltaica. Segundo os autores, "o regulamento foi eficaz em reduzir algumas barreiras naturais à entrada de sistemas fotovoltaicos." (SOUZA; MACHADO, 2016, p. 407).

Uma das principais vantagens na produção de energia elétrica por energia solar no local de consumo, em relação a qualquer outra fonte ecológica e energética, é a viabilidade econômica quanto à redução no consumo de eletricidade da rede de transmissão e distribuição de energia (PAINES; VIGNOCHI; POSSAMAI, 2018). Conforme os autores, os principais desafios não técnicos envolvem questões de conscientização, políticas governamentais e opções de financiamento.

Fator importante para o desempenho do gerador é o ângulo de incidência da luz solar, que depende da localização geográfica da edificação e condições do clima (MELO et al., 2017). A radiação incidente em uma superfície pode ocorrer por dois processos. O primeiro é a 
radiação de feixe direto que vem do Sol e pode ser calculado matematicamente. $O$ segundo componente é a radiação difusa, que surge da dispersão da luz na atmosfera em razão da reflexão e absorção por átomos de compostos do ar. Portanto, para o uso da tecnologia fotovoltaica existe a necessidade de avaliação quanto à viabilidade na produção para cada instalação do sistema.

Especialmente na Engenharia Civil, componentes na estrutura física da edificação passam a ser um critério importante para o uso da tecnologia. Com este intuito, o objetivo deste estudo foi realizar uma análise comparativa entre dois estabelecimentos de ensino públicos estaduais localizados na cidade de Concórdia, relacionados à viabilidade técnica e econômica para a implantação de uma usina de geração de energia elétrica solar fotovoltaica.

Quanto à viabilidade econômica, estudos realizados desde 2015 na Universidade do Contestado, Campus de Concórdia, com diferentes procedimentos metodológicos, demostraram viabilidade econômica para a instalação da tecnologia (BIFFl; REIS, 2019). Nas pesquisas, o payback foi obtido com cálculos em planilhas eletrônicas, utilizando modelo também utilizado na Índia (NAYAK et al., 2014). Os resultados no Brasil e na Índia demonstraram viabilidade econômica para o uso da tecnologia nas situações de contexto onde foram aplicadas. Pesquisa realizada em posto de combustível demostrou que o sistema é economicamente viável, com retorno financeiro em menos de seis anos (BIFFI; REIS, 2019). O que difere nesta pesquisa é o fato de serem edificações públicas, empreendimentos em que o custo da energia possui taxa reduzida em relação ao custo médio local.

A partir das considerações dissertadas, com foco na sustentabilidade e eficiência energética nos empreendimentos e com os dados coletados em dois estabelecimentos públicos, a pesquisa foi realizada com o objetivo de investigar a viabilidade técnica e econômica, a partir de análise comparativa com dados de uma escola urbana e uma rural, a fim de propor a instalação de usina solar fotovoltaica nesses empreendimentos.

\section{METODOLOGIA}

Inicialmente foram identificadas as escolas que fariam parte da amostra, representando a rede pública de Concórdia. Esses estabelecimentos foram avaliados com dados qualitativos e quantitativos coletados junto à instituição, empresa distribuidora de energia elétrica e empresas fornecedoras da tecnologia, a fim de possibilitar a realização da pesquisa. Em cada uma das escolas públicas selecionadas para o estudo de caso (rural e urbana) foi aplicada a seguinte metodologia:
Avaliação do potencial de consumo: para a realização do estudo, foram coletados dados dos custos mensais e anual no consumo de energia elétrica dos estabelecimentos (escolas), utilizando pesquisa documental (análise das faturas mensais de 2018).

\subsection{Avaliação da eficiência energética}

Em cada um dos estabelecimentos foi realizado um levantamento sobre os pontos de consumo, como quantidade de lâmpadas, equipamentos de climatização do ambiente, TV e outros equipamentos. Essa etapa foi realizada em atividade de ensino, com a participação de estudantes do $9^{\circ}$ ano e Ensino Médio. A participação dos estudantes na avaliação do consumo nas salas de aula e demais dependências das escolas ocorreu sem que houvesse a participação direta dos estudantes nos dados coletados. Por conta disso, o projeto não foi submetido ao Comitê de Ética na Plataforma Brasil. O autor principal desta pesquisa é professor de Matemática nessas duas instituições, critério utilizado na escolha dos estabelecimentos. A avaliação dos pontos de consumos foi importante para relacionar a eficiência e sustentabilidade energética em cada estabelecimento e avaliar o consumo anual.

\subsection{Avaliação da viabilidade na instalação fotovoltaica}

Levantamento de dados com empresa fornecedora da tecnologia fotovoltaica, a qual realizou uma simulação para estudo da viabilidade econômica e orçamentária para cada um dos estabelecimentos. A simulação foi realizada para avaliar a viabilidade de instalação de sistema de geração fotovoltaica, produção de energia e a viabilidade econômica para a futura instalação do sistema fotovoltaico.

Para a realização dos cálculos do tempo de retorno do investimento - Payback e Rentabilidade, como realizado em estudos anteriores (NAYAK et al., 2014), foram utilizadas as seguintes equações:

$$
\begin{gathered}
\text { PAYBACK (meses) }\left[\frac{\text { (investimento } \mathrm{R} S)}{\left(\text { Energia Gerada } \frac{\mathrm{kw}}{\mathrm{més}} \times \text { valor da tarifa }(\mathrm{R} \$)\right)}\right] \\
\text { Rentabilidade }=\left[\frac{\mathrm{e}}{\text { (Economia) }}\right]
\end{gathered}
$$

\subsection{Avaliação de estrutura física para instalação fotovoltaica}

Foi realizada a avaliação da edificação quanto às condições físicas, localização geográfica e condições meteorológicas (como a incidência e curva solar), a fim de avaliar 
as características do local, bem como o ambiente mais adequado às instalações. Com os dados dos itens anteriores, foi realizado o comparativo entre os dois sistemas propostos, identificando o mais favorável. Os resultados encontrados para cada projeto foram comparados entre os dois estabelecimentos e com os dados coletados em outras etapas da pesquisa.

\section{RESULTADOS DA PESQUISA}

\subsection{Perfil dos empreendimentos}

\subsubsection{Estabelecimento $A$}

O estabelecimento $A$ é uma escola da rede pública localizada no centro de Concórdia, nas coordenadas geográficas na Latitude: $27^{\circ} 13^{\prime} 59$, Longitude: $52^{\circ} 2^{\prime} 3$ e Azimute: 321.23 (Figura 1). A edificação é de alvenaria, com telha asbesto-cimento ou fibrocimento, em bom estado de conservação. Quanto ao posicionamento dos telhados, possui áreas para o norte, proporcionando condições de insolação para instalação da tecnologia. A avaliação da estrutura física do empreendimento contempla 12 salas de aula e 10 salas de apoio que abrigam outros setores da escola, para atender, em média, de 690 a 704 alunos, em

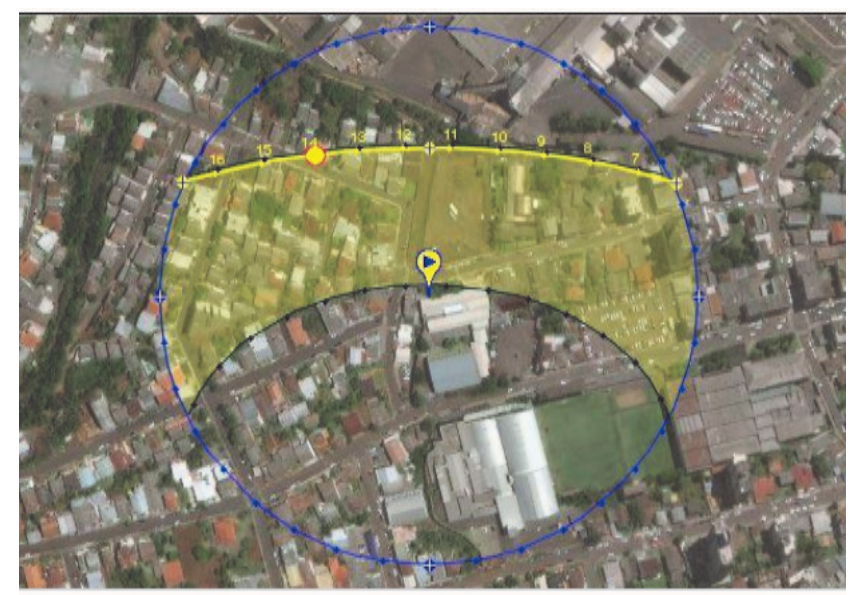

Figura 1 - Localização geográfica e curva solar na Escola A

Fonte: autores, a partir de imagens fornecidas pelo software SunEarthTools.com.

\subsubsection{Estabelecimento $B$}

O estabelecimento $B$ também é uma escola da rede pública, localizada em distrito (interior do município) de Concórdia, com as coordenadas de Latitude: $27^{\circ} 18^{\prime} 14$, Longitude: $51^{\circ} 59^{\prime} 43$ e Azimute 321.22. Com um total de 12 salas, atende um total de 227 alunos, em dois turnos (matutino e vespertino). Como no empreendimento anterior, a edificação é de alvenaria, com telha asbesto-cimento ou fibrocimento, em bom estado de conservação. Também possui áreas para o norte, proporcionando condições de insolação para a instalação da tecnologia.

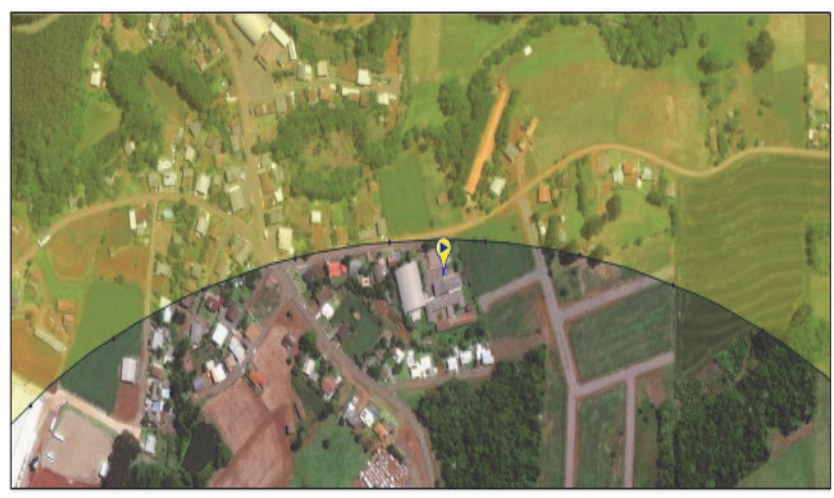

Figura 2 - Localização geográfica e curva solar na Escola B

Fonte: autores, a partir de imagens fornecidas pelo software SunEarthTools.com.

\subsection{Avaliação comparativa para o potencial de consumo}

Os dados coletados sobre o consumo de energia demonstraram diferenças significativas entre os dois estabelecimentos. $\mathrm{O}$ estabelecimento $\mathrm{B}$ apresentou valores de consumo (em kWh), em média, 50\% menor que o estabelecimento $A$, ao longo do período analisado (Figura 3). A diferença se justifica em razão do menor número de salas e de estudantes.

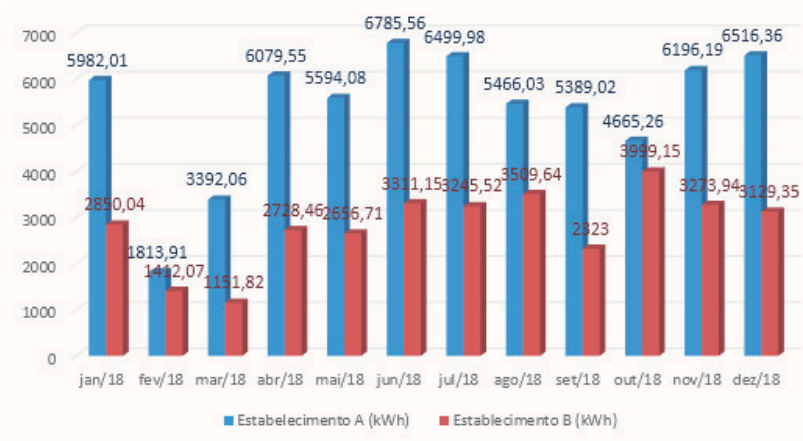

Figura 3 - Comparativo entre os consumos para os estabelecimentos A e B Fonte: autores.

Quanto ao valor médio por kWh consumido (em reais), no período de janeiro a dezembro de 2018, foi de aproximadamente $\mathrm{R} \$ \mathbf{0}, 57$, para ambos os estabelecimentos estudados. Em relação ao consumo médio mensal no estabelecimento A foi de $5364,80 \mathrm{~kW} / \mathrm{mês}$ e no estabelecimento B foi de 2798,90 kW/mês (Tabela 1).

\begin{tabular}{|l|l|l|l|l|}
\hline & \multicolumn{2}{|l|}{ Estabelecimento A } & \multicolumn{2}{l|}{ Estabelecimento B } \\
\hline Tipo de Conexão & Trrifásico & & Trifásico & \\
\hline Disjuntor & 50 & Ampères & 50 & Ampères \\
\hline
\end{tabular}




\begin{tabular}{|l|l|l|l|l|}
\hline Média de consumo & 5364,8 & $\begin{array}{l}\mathrm{kWh} / \\
\mathrm{mês}\end{array}$ & 2798,9 & $\mathrm{kWh} / \mathrm{mês}$ \\
\hline Demanda/ano & 64377 & $\begin{array}{l}\mathrm{kWh} / \\
\text { anual }\end{array}$ & 33586 & $\mathrm{kWh} / \mathrm{ano}$ \\
\hline $\begin{array}{l}\text { Custo da tarifa } \\
\text { por Wh }\end{array}$ & 0,57 & $\mathrm{reais}$ & 0,57 & $\mathrm{reais}$ \\
\hline $\begin{array}{l}\text { Sistema } \\
\text { necessário }\end{array}$ & 45,09 & $\mathrm{kWp}$ & 23,53 & $\mathrm{kWp}$ \\
\hline $\begin{array}{l}\text { Consumo a } \\
\text { atender }\end{array}$ & 5364,8 & $\mathrm{kWh} /$ & 2798,9 & $\mathrm{kWh} / \mathrm{mês}$ \\
\hline Sistema orçado & 45,9 & $\mathrm{kWp}$ & 25,5 & $\mathrm{kWp}$ \\
\hline $\begin{array}{l}\text { Número de } \\
\text { Módulos }\end{array}$ & 135 & $\mathrm{unidades}$ & 75 & $\mathrm{unidades}$ \\
\hline $\begin{array}{l}\text { Área de Instalação } \\
\text { Én }\end{array}$ & 270 & $\mathrm{~m}{ }^{2}$ & 150 & $\mathrm{~m} 2$ \\
\hline $\begin{array}{l}\text { Estimativa de } \\
\text { Geração Ano }\end{array}$ & 65545,2 & $\mathrm{~kW} / \mathrm{ano}$ & 36414 & $\mathrm{~kW} / \mathrm{anno}$ \\
\hline $\begin{array}{l}\text { Estimativa de } \\
\text { Geração Mês }\end{array}$ & 5462,1 & $\mathrm{~kW} / \mathrm{mês}$ & 3034,5 & $\mathrm{~kW} / \mathrm{mês}$ \\
\hline
\end{tabular}

Tabela 1 - Dados de dimensionamento - empreendimentos A e B Fonte: autores, a partir das informações da empresa fornecedora da tecnologia.

Ao simular os dados para atender à demanda de 45,09 kW de potência no empreendimento $A$, são necessários 135 painéis de 340 Watts, sendo necessária uma superfície com aproximadamente $270 \mathrm{~m}^{2}$ de área. Para atender à demanda de $23,53 \mathrm{~kW}$ de potência, o empreendimento $B$ necessitará de 75 painéis de 340 Watts, em uma superfície de aproximadamente $150 \mathrm{~m}^{2}$ de área.

\subsection{Sustentabilidade no consumo de energia elétrica nas edificações}

A fim de avaliar a sustentabilidade no uso da eletricidade em cada um dos estabelecimentos, em uma atividade de aula, com a participação dos estudantes de ensino básico (9॰ ano e ensino médio), foram realizadas avaliações da potência consumida. Desse modo, foi verificada a quantidade de lâmpadas e equipamentos (computacionais, de climatização e outros), bem como as potências, tempo de uso e consumo médio mensal. Essa atividade foi desenvolvida nas aulas de Ciências e Matemática, com a intenção de integrar Ciência, Tecnologia, Engenharia e Matemática, como sugere o movimento STEM. A participação dos estudantes nessa etapa teve como propósito construir junto com eles as concepções de sustentabilidade energética.

Os dados coletados e registrados em tabelas por estudantes foram computados e apresentados na Tabela 2, a seguir. Os resultados demonstram que os equipamentos consomem parcela significativa da demanda, o que justifica a variação na produção de energia durante o período analisado, em razão da necessidade de uso nos equipamentos.

\begin{tabular}{|l|l|l|l|l|}
\hline & \multicolumn{2}{|l|}{ Empreendimento A } & \multicolumn{2}{l|}{ Empreendimento B } \\
\cline { 2 - 5 } & kW/mês & Custo & kW/mês & Custo \\
\hline Iluminação das salas & 2330,9 & 1319,71 & 1382,4 & 782,64 \\
\hline Iluminação do ginásio & 10,4 & 942,12 & 12 & 543,53 \\
\hline Demais consumos & 3023,7 & 1711,95 & 1404,83 & 795,39 \\
\hline
\end{tabular}

Tabela 2 - Resultado da avaliação de consumo em cada escola Fonte: 0 s autores.

\subsection{Características do sistema fotovoltaico proposto}

O sistema fotovoltaico é composto por geradores, inversor de corrente e acessórios (cabos, conectores, suporte e String Box). A seguir são apresentadas as características do material proposto para cada um dos estabelecimentos.

Gerador fotovoltaico: Módulos fotovoltaicos são compostos por células de silício policristalino, responsáveis pela transformação da luz do Sol em eletricidade, com eficiência de $18,1 \%$ na transformação da radiação solar em eletricidade. Para que o sistema atinja a capacidade de 45,9 kWp são necessários 135 módulos fotovoltaicos de 340 Watts no empreendimento A. E para 25,5 kWp são necessários 75 módulos fotovoltaicos de 340 Watts no empreendimento B. Os módulos possuem garantia de 10 anos contra defeito de fabricação e garantem produção por um período de 25 anos, com no mínimo $80 \%$ de eficiência.

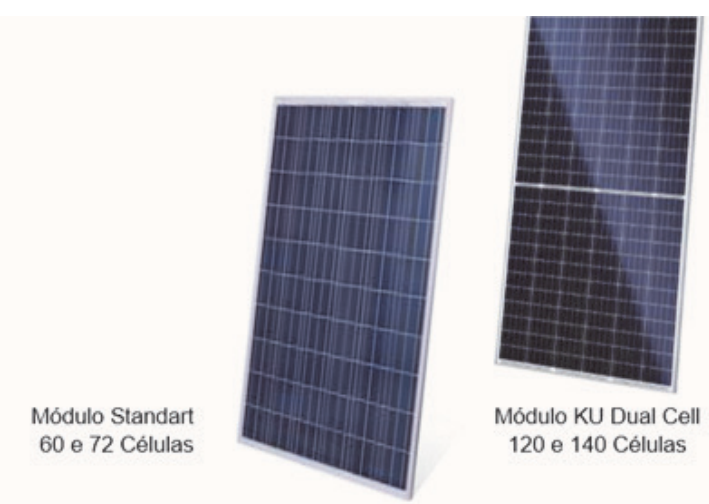

Figura 4 - Especificações do gerador

Fonte: os autores, a partir do relatório do orçamento realizado junto à empresa fornecedora da tecnologia.

Inversor de corrente: Responsável por transformar a corrente contínua (CC) gerada no painel em corrente alternada $(A C)$, para ser compartilhada com a rede elétrica convencional e uso na edificação. Para o projeto do empreendimento $A$, a opção da empresa foi o inversor marca A de 36 kW (Figura 5a), para tensão máxima de 1100 V, com alta velocidade, precisão no monitoramento e captação de energia. Com eficiência de $98 \%$, é proporcionado 5 anos de garantia do fabricante. 


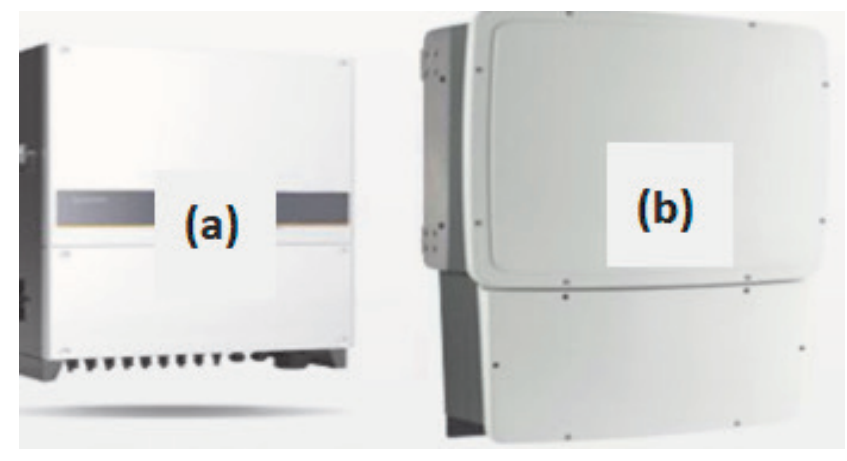

Figura 5 - Inversores de corrente contínua para corrente alternada.

Fonte: 0 s autores, a partir das informações da empresa fornecedora da tecnologia. Na figura a) 0 inversor proposto no estabelecimento $A$ e na figura $b$ ) 0 inversor proposto no estabelecimento $B$

Para o projeto do estabelecimento B a opção da empresa foi um inversor marca B (Figura 5b), com características, eficiência e garantia, como no inversor do projeto anterior, porém com potência nominal de $20 \mathrm{~kW}$, tensão de entrada de 320V e de saída 320V - 480V. A diferenciação pelo inversor ocorreu em razão da necessidade de potência instalada e viabilidade econômica, sugerindo marcas diferentes.

Acessório para instalações: Ambos os projetos sugerem cabos de $6 \mathrm{~mm}$, específicos para ligar os painéis aos inversores, suporte para fixação dos módulos, sendo barras de alumínio ultra light, parafusos, terminais intermediários e finais.

\subsection{Tempo de retorno dos investimentos: payback e rentabilidade}

O tempo de retorno do investimento em energia solar (payback) representa o tempo necessário para que ocorra o retorno dos investimentos iniciais. Foram considerados os custos de instalação do sistema e a economia mensalmente proporcionada com a energia gerada na usina solar fotovoltaica. Desse modo, o payback e rentabilidade do empreendimento A foi obtido considerando um investimento inicial de $\mathrm{R} \$ 142.655,42$ e produção energética de 5462,10 kW/mês, aplicando o seguinte cálculo (Eq. 03 e 04):

$$
\begin{gathered}
\text { PAYBACK A (meses) }=\left[\frac{(\mathrm{R} \$ 142.655,42)}{\left(5462,10 \frac{\mathrm{kw}}{m \mathrm{es}} \times \mathrm{R} \$ 0,57\right)}\right] \\
\text { Rentabilidade }=\left[\frac{\mathrm{e} \$(3) .110,38)}{(\mathrm{R} \$ 142.655,42)}\right]=26 \% \text { (4) }
\end{gathered}
$$

Desse modo a rentabilidade do sistema proposto no empreendimento A foi de $26 \%$. E para o investimento B, onde o investimento inicial ficou em R\$ 89.293,98, para um sistema que gera $3034,50 \mathrm{~kW} / \mathrm{mês}$, o payback e rentabilidade do empreendimento também foi aplicado o mesmo cálculo (Eq. 05 e 06).

$$
\begin{gathered}
\text { PAYBACK B (meses) }=\left[\frac{(\mathrm{R} \$ 89.293,98)}{\left(3034,50 \frac{k w}{m e s} \times R \$ 0.57\right)}\right] \text { (5) } \\
\text { Rentabilidade }=\left[\frac{\mathrm{R} \$ 20.616,88)}{(\mathrm{R} \$ 89.293,98)}\right]=23 \%(6)
\end{gathered}
$$

Portanto, o investimento inicial do projeto A é significativamente maior que para o projeto $B$, porém durante o período de 4 anos ocorre o retorno do investimento para ambos os projetos. O gráfico (Figura 6) para o tempo de retorno demonstra que no quarto ano os projetos apresentam valores semelhantes a serem resgatados desde o investimento inicial. A tendência de recuperação maior do investimento do projeto $A$ indica que tal projeto apresenta eficiência superior ao projeto $B$.

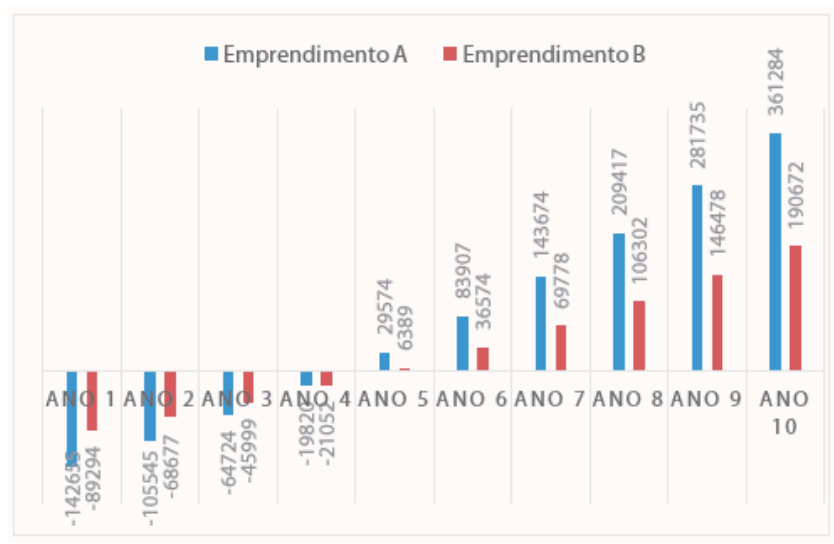

Figura 6 - Tempo de retorno do capital investido no projeto Fonte: 0 s autores.

No decorrer do período analisado essa tendência de eficiência demonstra ser significativamente maior no projeto $A$ em relação ao projeto $B$. Cabe ressaltar que os valores utilizados para a obtenção do gráfico se referem ao retorno dos valores financeiros (em reais), para cada ano após a instalação do projeto, com base nos orçamentos e tarifas para junho de 2019. O tempo de retorno poderá obter variações, a partir de mudanças no custo dos equipamentos, mudanças nos valores tributários e demais encargos financeiros. Devido ao fato que a tecnologia utilizada nesse sistema ser importada e seus custos apresentados em moeda internacional (\$), é possível que haja oscilações ao logo do tempo.

\section{CONCLUSÕES}

Edificações para uso em hospitais, supermercados, prédios públicos e empreendimentos comerciais geralmente possuem grandes áreas de cobertura e alto consumo de energia elétrica, fatores que interferem significativamente na viabilidade de projetos fotovoltaicos. O estudo 
objetivou analisar a viabilidade econômica do uso da energia solar fotovoltaica como alternativa de redução de custos nas faturas de energia elétrica em dois estabelecimentos públicos (escolas) localizados no município de Concórdia, Santa Catarina.

Nesse sentido, a pesquisa surge com a opção do uso da energia elétrica produzida por intermédio de fontes renováveis e infinitas, como a solar, a fim de contribuir para a sustentabilidade energética e econômica para os empreendimentos públicos (escolas). Essa possibilidade permite, ainda, promover junto aos estudantes de educação básica o uso sustentável de energia elétrica. Ações educacionais como essa podem oportunizar o desenvolvimento econômico de modo não linear, como sugere a economia circular.

Quanto ao estudo de caso nos empreendimentos contemplados na pesquisa, a análise de viabilidade econômica demonstrou ser viável à implantação da tecnologia, tanto no empreendimento A quanto no B. Cabe destacar que ao se tratar de instituição pública a energia é fornecida a valores subsidiados, ainda assim os resultados demonstraram que a instalação do sistema proposto pela empresa é viável. Para os cenários analisados, o tempo de retorno dos investimentos iniciais é inferior a cinco anos, somente considerando o custo da energia.

Por fim, conclui-se que a escolha da energia solar como fonte de energia elétrica pode diminuir os custos com o consumo de energia elétrica junto à concessionária e aumentar a sustentabilidade para os empreendimentos escolares, tornando-se uma opção para órgãos públicos. Ações desse tipo acabam proporcionando, em pouco tempo, economia em energia elétrica nos estabelecimentos educacionais e, consequentemente, ganhos para os cofres públicos e para uma educação em prol da sustentabilidade e preservação de recursos ambientais.

\section{REFERÊNCIAS}

BIFFI, E.; REIS, M. A. F. Estudo de viabilidade de usina solar fotovoltaica em posto de combustível na cidade de Ipumirim. VII Encontro de Sustentabilidade em Projeto. Anais...Florianópolis: UFSC, 2019Disponível em: <http://ensus2019.paginas. ufsc.br/anais-ensus-2019/>. Acesso em: 10 maio. 2019 CARVALHO, F. I. A. DE; ABREU, M. C. S. DE; CORREIA NETO, J. F. Financial Alternatives to Enable Distributed Microgeneration Projects With Photovoltaic Solar Power. RAM. Revista de Administração Mackenzie, v. 18, n. 1, p. 120-147, fev. 2017.

DARK, M. L. A photovoltaics module for incoming science, technology, engineering and mathematics undergraduates. Physics Education, v. 46, n. 3, p. 303-308, maio 2011.

KORHONEN, J.; HONKASALO, A.; SEPPÄLÄ, J. Circular Economy: The Concept and its Limitations. Ecological Economics, v. 143, n. January, p. 37-46, 2018.

MELO, A. G. et al. Development of a closed and open loop solar tracker technology. Acta Scientiarum. Technology, v. 39, n. 2, p. 177, 15 maio 2017.

NAYAK, S. et al. Energy matrices analysis of hybrid PVT greenhouse dryer by considering various silicon and non-silicon PV modules. International Journal of Sustainable Energy, v. 33, n. 2, p. 336-348, 2014.

PAINES, P. DE A.; VIGNOCHI, L.; POSSAMAI, O. Simulação de sistema fotovoltaico para o setor comercial. Exacta, v. 16, n. 3, p. 17-30, 27 set. 2018.

REIS, M. A. F. Efeito fotoelétrico na produção e transformação da luz: investigação do uso de uma proposta didática para o ensino de física em cursos de Engenharia. Canoas: ULBRA, 2019.

REIS, M. A. F.; SERRANO, A. Pesquisa bibliográfica em historicidade, conceitos e contextos na produção e transformação da luz com a teoria quântica. Acta Scientiae, v. 19, n. 3, p. 493-516, 2017.

SALAMONI, I. T.; RÜTHER, R.; ZILLES, R. Uma oportunidade para o desenvolvimento da indústria fotovoltaica no Brasil: eletricidade solar para os telhados. Parcerias SOUZA, A. C. G. DE; MACHADO, B. G. DE F. Sistemas fotovoltaicos de micro e minigeração distribuídas: mecanismos de incentivos e perspectivas de expansão à luz da Resolução Normativa no 482 / 2012 da Aneel. Caderno de Finanças Públicas, v. 1, n. 16, p. 387-410, 2016. 


\section{AUTORES}

ORCD: https://orcid.org/0000-0001-5319-138X

MARI AURORA FAVERO REIS, Dra. | Professora na Universidade do Contestado | Engenharia Civil | Concórdia, SC | Brasil | Correspondência para: R. Beira Rio, 48 - Bairro Jardim Tarumã, Xanxerê - SC, 89820-000 | E-mail: mariaurorafavero@gmail.com

ORCD: https://orcid.org/ 0000-0001-8153-5549

PAULO REIS JUNIOR, M.Sc. | Engenharia de Produção Faculdades (2014) | Mestrado Profissional em Engenharia Civil, Sanitária e Ambiental (PMPECSA - UnC) | Professor na Universidade do Contestado | Engenharia Civil | Concórdia, SC | Brasil | Correspondência para: R. Beira Rio, 48 - Bairro Jardim Tarumã, Xanxerê - SC, 89820-000| E-mail: sr.reis.paulo@gmail.com

ORCID: https://orcid.org/0000-0003-4082-893X

DIRCEU LORIVALDO PERIN | Graduação em Engenharia Civil na Universidade do Contestado. Licenciatura em Matemática, com pós-graduação em Educação Matemática (UnC). Professor de Matemática na Educação básica. | Correspondência para: dl-perin@bol.com.br

\section{COMO CITAR ESTE ARTIGO}

REIS, Mari Aurora Favero; REIS JUNIOR, Paulo; PERIN, Dirceu Lorivaldo. Sustentabilidade Energética em Escola Pública. MIX Sustentável, [S.I.], v. 6, n. 3, p. 37-44, jun. 2020. ISSN 24473073. Disponível em:<http://www.nexos.ufsc.br/index.php/mixsustentavel>. Acesso em: dia mês. ano. doi:https://doi. org/10.29183/2447-3073.MIX2020.v6.n3.37-44. 